\title{
MAPPING PROPERTIES FOR CONVOLUTIONS INVOLVING HYPERGEOMETRIC FUNCTIONS
}

\author{
J. A KIM and K. H. SHON
}

Received 4 March 2002

For $\mu \geq 0$, we consider a linear operator $L_{\mu}: A \rightarrow A$ defined by the convolution $f_{\mu} * f$, where $f_{\mu}=(1-\mu) z_{2} F_{1}(a, b, c ; z)+\mu z\left(z_{2} F_{1}(a, b, c ; z)\right)^{\prime}$. Let $\varphi^{*}(A, B)$ denote the class of normalized functions $f$ which are analytic in the open unit disk and satisfy the condition $z f^{\prime} / f \prec(1+A z) / 1+B z,-1 \leq A<B \leq 1$, and let $R_{\eta}(\beta)$ denote the class of normalized analytic functions $f$ for which there exits a number $\eta \in(-\pi / 2, \pi / 2)$ such that $\operatorname{Re}\left(e^{i \eta}\left(f^{\prime}(z)-\beta\right)\right)>0,(\beta<1)$. The main object of this paper is to establish the connection between $R_{\eta}(\beta)$ and $\varphi^{*}(A, B)$ involving the operator $L_{\mu}(f)$. Furthermore, we treat the convolution $I=\int_{0}^{z}\left(f_{\mu}(t) / t\right) d t * f(z)$ for $f \in R_{\eta}(\beta)$.

2000 Mathematics Subject Classification: 30C45.

1. Introduction. Let $A$ denote the class of functions of the form

$$
f(z)=z+\sum_{n=2}^{\infty} a_{n} z^{n}
$$

which are analytic in the open unit disk $U=\{z:|z|<1\}$ and $S$ denotes the subclass of functions in $A$ which are univalent in $U$. Moreover, let $S^{*}(\alpha)$ and $K(\alpha)$ be the subclasses of $S$ consisting, respectively, of functions which are starlike of order $\alpha$ and convex of order $\alpha$, where $0 \leq \alpha<1$ in $U$. Clearly, we have $S^{*}(\alpha) \subseteq S^{*}(0)=S^{*}$, where $S^{*}$ denotes the class of functions in $A$ which are starlike in $U$ and $K(\alpha) \subseteq K(0)=K$, where $K$ denotes the class of functions in $A$ which are convex in $U$, and we mention the well-known inclusion chain $K \subset S^{*}(1 / 2) \subset S^{*} \subset S$. For the analytic functions $g$ and $h$ on $U$ with $g(0)=h(0), g$ is said to be subordinate to $h$ if there exists an analytic function $w$ on $U$ such that $w(0)=0,|w(z)|<1$, and $g(z)=h(w(z))$ for $z \in U$. We denote this subordinated relation by

$$
g \prec h \quad \text { or } \quad g(z) \prec h(z) \quad(z \in U) \text {. }
$$

For $-1 \leq A<B \leq 1$, a function $p$, which is analytic in $U$ with $p(0)=1$, is said to belong to the class $P(A, B)$ if

$$
p(z) \prec \frac{1+A z}{1+B z} \quad(z \in U) .
$$


The above condition means that $p$ takes the values in the disk with a center $(1-A B) /\left(1-B^{2}\right)$ and a radius $|A-B| /\left(1-B^{2}\right)$. The boundary circle cuts the real axis at the points $(1+A) /(1+B)$ and $(1-A) /(1-B)$. A function $f \in A$ is said to be in $\varphi^{*}(A, B)$ if $z f^{\prime} / f \in P(A, B)$, and in $K(A, B)$ if $z f^{\prime} \in \varphi^{*}(A, B)$. The class $\varphi^{*}(A, B)$ was introduced by N. Shukla and P. Shukla [4]. Also, Janowski [2] introduced the class $P(A, B)$. For the fixed natural number $n$, the subclass $P_{n}(A, B)$ of $P(A, B)$ containing functions $p$ of the form $p(z)=1+p_{n} z^{n}+\cdots$, $z \in U$, was defined by Stankiewicz and Waniurski [7]. In addition, Stankiewicz and Trojnar-Spelina [6] investigated a function $p(z)=1-p_{n} z^{n}-\cdots$ belongs to the class $R(n, A, B)$, where $A \in R$ and $B \in[0,1]$ if $p(z) \prec(1+A z) /(1-B z)$. Let $R_{\eta}(\beta)$ denote the class of functions $f \in A$ for which there exists a number $\eta \in(-\pi / 2, \pi / 2)$ such that

$$
\operatorname{Re}\left[e^{i \eta}\left(f^{\prime}(z)-\beta\right)\right]>0 \quad(z \in U, \beta<1) .
$$

Clearly, we have $R_{\eta}(\beta) \subset S(0 \leq \beta<1)$. Furthermore, if a function $f$ of the form (1.1) belongs to the class $R_{\eta}(\beta)$, then

$$
\left|a_{n}\right| \leq \frac{2(1-\beta) \cos \eta}{n} \quad(n \in N \backslash\{1\})
$$

The class $R_{\eta}(\beta)$ was studied by Kanas and Srivastava [3].

The hypergeometric function ${ }_{2} F_{1}(a, b, c ; z)$ is given as a power series, converging in $U$, in the following way

$$
{ }_{2} F_{1}(a, b, c ; z)=\sum_{n=0}^{\infty} \frac{(a)_{n}(b)_{n}}{(c)_{n}(1)_{n}} z^{n},
$$

where $a, b$, and $c$ are complex numbers with $c \neq 0,-1,-2, \ldots$, and $(\lambda)_{n}$ denotes the Pochhammer symbol (or the generalized factorial since $(1)_{n}=n$ !) defined, in terms of the Gamma function $\Gamma$, by

$$
\begin{aligned}
(\lambda)_{n}: & : \frac{\Gamma(\lambda+n)}{\Gamma(\lambda)} \\
& = \begin{cases}1 & \text { if } n=0, \\
\lambda(\lambda+1) \cdots(\lambda+n-1) & \text { if } n \in N=\{1,2, \ldots\} .\end{cases}
\end{aligned}
$$

Note that ${ }_{2} F_{1}(a, b, c ; z)$, for $a=c$ and $b=1$ (or, alternatively, for $a=1$ and $b=c$ ), reduces to the relatively more familiar geometric function. We also 
note that ${ }_{2} F_{1}(a, b, c ; 1)$ converges for $\operatorname{Re}(c-a-b)>0$ and is related to the Gamma functions by

$$
{ }_{2} F_{1}(a, b, c ; 1)=\frac{\Gamma(c) \Gamma(c-a-b)}{\Gamma(c-a) \Gamma(c-b)} .
$$

The Hadamard product (or convolution) of two power series $f(z)=\sum_{n=0}^{\infty} a_{n} z^{n}$ and $g(z)=\sum_{n=0}^{\infty} b_{n} z^{n}$ is defined as the power series

$$
(f * g)(z)=\sum_{n=0}^{\infty} a_{n} b_{n} z^{n}
$$

N. Shukla and P. Shukla [4] studied the mapping properties of a function $f_{\mu}$ to be as given in

$$
f_{\mu}(z)=(1-\mu) z_{2} F_{1}(a, b, c ; z)+\mu z\left(z_{2} F_{1}(a, b, c ; z)\right)^{\prime} \quad(\mu \geq 0),
$$

and investigated the geometric properties of an integral operator of the form

$$
I(z)=\int_{0}^{z} \frac{f_{\mu}(t)}{t} d t .
$$

We now consider a linear operator $L_{\mu}: A \rightarrow A$ defined by

$$
L_{\mu}(f)=f_{\mu}(z) * f(z) .
$$

For $\mu=0$ in (1.12), $L_{\mu}(f)=\left[I_{a, b, c}(f)\right](z)$, which was introduced by Hohlov [1]. Also, Kanas and Srivastava [3], and Srivastava and Owa [5] showed that the operator $I_{a, b, c}(f)$ is the natural extensions of the Alexander, Libera, Bernardi, and Carlson-Shaffer operators. In this paper, we find a relation between $R_{\eta}(\beta)$ and $\varphi^{*}(A, B)$ involving the operator $L_{\mu}(f)$. Furthermore, we study to obtain some conditions for the starlikeness and convexity of the convolution of $I$ and $f$, which are given by (1.11) and (1.1), respectively, for $f \in R_{\eta}(\beta)$.

2. Main results. We make use of the following lemma.

LEMMA 2.1 [4]. Sufficient conditions for $f$ of the form (1.1) to be in $\varphi^{*}(A, B)$ and $K(A, B)$ are

$$
\begin{aligned}
& \sum_{n=2}^{\infty}[(1+B) n-(A+1)]\left|a_{n}\right| \leq B-A, \\
& \sum_{n=2}^{\infty} n[(1+B) n-(A+1)]\left|a_{n}\right| \leq B-A,
\end{aligned}
$$

respectively. 
THEOREM 2.2. Let $a>1, b>1$, and $c>a+b+1$. If $f \in R_{\eta}(\beta)$ and the inequality

$$
\begin{gathered}
\frac{\Gamma(c) \Gamma(c-a-b)}{\Gamma(c-a) \Gamma(c-b)}\left[(1+B)\left(1+\frac{\mu a b}{c-a-b-1}\right)-(A+1)\left(\mu-\frac{(\mu-1)(c-a-b)}{(a-1)(b-1)}\right)\right] \\
\leq(B-A)\left(\frac{1}{2(1-\beta) \cos \eta}+1\right)+\frac{(A+1)(\mu-1)(c-1)}{(a-1)(b-1)}
\end{gathered}
$$

is satisfied, then $L_{\mu}(f) \in \varphi^{*}(A, B)$.

Proof. By Lemma 2.1, it suffices to show that

$$
T_{1}:=\sum_{n=2}^{\infty}[(1+B) n-(A+1)]\left|\frac{(1+(n-1) \mu)(a)_{n-1}(b)_{n-1}}{(c)_{n-1}(1)_{n-1}} a_{n}\right| \leq B-A .
$$

Since $f \in R_{\eta}(\beta)$ and $\left|a_{n}\right| \leq 2(1-\beta) \cos \eta / n$. Hence,

$$
\begin{aligned}
& T_{1} \leq \sum_{n=2}^{\infty}[(1+B) n-(A+1)] \frac{(1+(n-1) \mu)(a)_{n-1}(b)_{n-1}}{(c)_{n-1}(1)_{n-1}} \frac{2(1-\beta) \cos \eta}{n} \\
& =2(1-\beta) \cos \eta\left\{(1+B)\left(\sum_{n=0}^{\infty} \frac{(a)_{n}(b)_{n}}{(c)_{n}(1)_{n}}-1\right)\right. \\
& -\frac{(A+1)(c-1)}{(a-1)(b-1)}\left(\sum_{n=0}^{\infty} \frac{(a-1)_{n}(b-1)_{n}}{(c-1)_{n}(1)_{n}}-1-\frac{(a-1)(b-1)}{c-1}\right) \\
& +\frac{(1+B) \mu a b}{c} \sum_{n=0}^{\infty} \frac{(a+1)_{n}(b+1)_{n}}{(c+1)_{n}(1)_{n}} \\
& -(A+1) \mu\left[\sum_{n=0}^{\infty} \frac{(a)_{n}(b)_{n}}{(c)_{n}(1)_{n}}-1\right. \\
& -\frac{c-1}{(a-1)(b-1)}\left(\sum_{n=0}^{\infty} \frac{(a-1)_{n}(b-1)_{n}}{(c)_{n}(1)_{n}}\right. \\
& \left.\left.\left.-1-\frac{(a-1)(b-1)}{c-1}\right)\right]\right\} \\
& =2(1-\beta) \cos \eta\left\{\frac { \Gamma ( c ) \Gamma ( c - a - b ) } { \Gamma ( c - a ) \Gamma ( c - b ) } \left[(1+B)\left(1+\frac{\mu a b}{c-a-b-1}\right)\right.\right. \\
& \left.+(A+1)\left(\mu-\frac{(\mu-1)(c-a-b)}{(a-1)(b-1)}\right)\right] \\
& \left.-\left[1+B-(A+1)\left(1-\frac{(\mu-1)(c-1)}{(a-1)(b-1)}\right)\right]\right\} \text {. }
\end{aligned}
$$

Now, this last expression is bounded above by $B-A$ if (2.2) holds. 
If we take $\mu=0, A=2 \alpha-1$, and $B=1$ in Theorem 2.2, we have the following corollary.

COROLlary 2.3. Let $a>1, b>1$, and $c>a+b+1$. If $f \in R_{\eta}(\beta)$ and the inequality

$$
\begin{aligned}
& \frac{\Gamma(c) \Gamma(c-a-b)}{\Gamma(c-a) \Gamma(c-b)}\left[1-\frac{\alpha(c-a-b)}{(a-1)(b-1)}\right] \\
& \quad \leq(1-\alpha)\left(\frac{1}{2(1-\beta) \cos \eta}+1\right)-\frac{\alpha(c-1)}{(a-1)(b-1)}
\end{aligned}
$$

is satisfied, then $z_{2} F_{1}(a, b, c ; z) * f \in S^{*}(\alpha)$.

If we take $\alpha=0, \beta=0$, and $\eta=0$ in Corollary 2.3, we get the following corollary.

COROLlary 2.4. Let $a>1, b>1$, and $c>a+b+1$. If $f \in S$, and the inequality

$$
\frac{\Gamma(c) \Gamma(c-a-b)}{\Gamma(c-a) \Gamma(c-b)} \leq \frac{3}{2}
$$

is satisfied, then $z_{2} F_{1}(a, b, c ; z) * f \in S^{*}$.

THEOREM 2.5. Let $a>0, b>0$, and $c>a+b+2$. If $f \in R_{\eta}(\beta)$, and the inequality

$$
\begin{aligned}
& \frac{\Gamma(c) \Gamma(c-a-b)}{\Gamma(c-a) \Gamma(c-b)}[B-A+((1+B)(1+2 \mu) \\
& \left.\quad-(A+1) \mu) \frac{a b}{c-a-b-1}+\frac{(1+B) \mu(a)_{2}(b)_{2}}{(c-a-b-2)_{2}}\right] \\
& \quad \leq(B-A)\left(\frac{1}{2(1-\beta) \cos \eta}+1\right)
\end{aligned}
$$

is satisfied, then $L_{\mu}(f) \in K(A, B)$.

Proof. The proof follows from Lemma 2.1. Using the method of the proof of Theorem 2.2, we omit the details involved.

For $\mu=0, A=2 \alpha-1$, and $B=1$, Theorem 2.5 yields the following corollary. 
COROLLARY 2.6. Let $a>0, b>0$, and $c>a+b+2$. If $f \in R_{\eta}(\beta)$ and the inequality

$$
\frac{\Gamma(c) \Gamma(c-a-b)}{\Gamma(c-a) \Gamma(c-b)}\left[1-\alpha+\frac{a b}{c-a-b-1}\right] \leq(1-\alpha)\left(\frac{1}{2(1-\beta) \cos \eta}+1\right)
$$

is satisfied, then $z_{2} F_{1}(a, b, c ; z) * f \in K(\alpha)$.

For $\alpha=0, \beta=0$, and $\eta=0$, Corollary 2.6 yields the following corollary.

COROLlary 2.7. Let $a>0, b>0$, and $c>a+b+1$. If $f \in S$ and the inequality

$$
\frac{\Gamma(c) \Gamma(c-a-b)}{\Gamma(c-a) \Gamma(c-b)}\left[1+\frac{a b}{c-a-b-1}\right] \leq \frac{3}{2}
$$

is satisfied, then $z_{2} F_{1}(a, b, c ; z) * f \in K$.

In our next theorems, we find the sufficient conditions for $I * f$ to be in $\varphi^{*}(A, B)$ and $K(A, B)$. From the definition of $I$ given by (1.11), we obtain

$$
I(z)=z+\sum_{n=2}^{\infty} \frac{((1-\mu)+n \mu)(a)_{n-1}(b)_{n-1}}{(c)_{n-1}(1)_{n}} z^{n} \quad(\mu \geq 0, z \in U) .
$$

THEOREM 2.8. Let $a>1, b>1$, and $c>a+b$. If $f \in R_{\eta}(\beta)$ and the inequality

$$
\begin{gathered}
(1+B-(A+1) \mu)_{2} F_{1}(a, b, c ; 1)-(A+1)(1-\mu)_{4} F_{3}(a, b, 1,1, c, 2,2 ; 1) \\
\leq(B-A)\left(\frac{1}{2(1-\beta) \cos \eta}+1\right)
\end{gathered}
$$

is satisfied, then $I * f \in \varphi^{*}(A, B)$.

Proof. By Lemma 2.1, it satisfies to show that

$$
T_{2}:=\sum_{n=2}^{\infty}((1+B) n-(A+1))\left|\frac{(1-\mu+n \mu)(a)_{n-1}(b)_{n-1}}{(c)_{n-1}(1)_{n}} a_{n}\right| \leq B-A .
$$


Suppose that $f \in R_{\eta}(\beta)$. Then by (1.5) we observe that

$$
\begin{aligned}
T_{2} \leq \sum_{n=2}^{\infty}((1+B) n- & (A+1)) \frac{(1-\mu+n \mu)(a)_{n-1}(b)_{n-1}}{(c)_{n-1}(1)_{n}} \frac{2(1-\beta) \cos \eta}{n} \\
=2(1-\beta) \cos \eta\{ & ((1+B)(1-\mu)-(A+1) \mu) \sum_{n=2}^{\infty} \frac{(a)_{n-1}(b)_{n-1}}{(c)_{n-1}(1)_{n}} \\
& -(A+1)(1-\mu) \sum_{n=2}^{\infty} \frac{(a)_{n-1}(b)_{n-1}}{(c)_{n-1}(1)_{n+1}} \\
& +(1+B) \mu \sum_{n=2}^{\infty} \frac{\left.(a)_{n-1}(b)_{n-1}\right\}}{\left.(c)_{n-1}(1)_{n-1}\right\}} \\
=2(1-\beta) \cos \eta\{ & ((1+B)(1-\mu)-(A+1) \mu)\left(\frac{c-1}{(a-1)(b-1)}+{ }_{2} F_{1}(a, b, c ; 1)\right) \\
& -(A+1)(1-\mu)_{4} F_{3}(a, b, 1,1, c, 2,2 ; 1) \\
& +(1+B) \mu_{2} F_{1}(a, b, c ; 1) \\
& \left.-\left[((1+B)(1-\mu)-(A+1) \mu) \frac{c-1}{(a-1)(b-1)}+B-A\right]\right\}
\end{aligned}
$$

$\leq B-A$

by (2.11). This completes the proof.

Taking $\mu=0, A=2 \alpha-1$, and $B=1$ in Theorem 2.8, we see the following corollary.

COROLLARY 2.9. Let $a>1, b>1$, and $c>a+b$. If $f \in R_{\eta}(\beta)$ and the inequality

$$
{ }_{2} F_{1}(a, b, c ; 1)-\alpha_{4} F_{3}(a, b, 1,1, c, 2,2 ; 1) \leq(1-\alpha)\left(\frac{1}{2(1-\beta) \cos \eta}+1\right)
$$

is satisfied, then $\int_{0}^{z}{ }_{2} F_{1}(a, b, c ; t) d t * f \in S^{*}(\alpha)$.

Taking $\alpha=0, \beta=0$, and $\eta=0$ in Corollary 2.9, we get the following corollary.

COROLlary 2.10. Let $a>1, b>1$, and $c>a+b$. If $f \in S$ and the inequality

$$
\frac{\Gamma(c) \Gamma(c-a-b)}{\Gamma(c-a) \Gamma(c-b)} \leq \frac{3}{2}
$$

is satisfied, then $\int_{0}^{z} F_{1}(a, b, c ; t) d t * f \in S^{*}$. 
THEOREM 2.11. Let $a>1, b>1$, and $c>a+b+1$. If $f \in R_{\eta}(\beta)$ and the inequality

$$
\begin{aligned}
& \frac{\Gamma(c) \Gamma(c-a-b)}{\Gamma(c-a) \Gamma(c-b)}\left[(1+B)\left(1+\frac{\mu a b}{c-a-b-1}\right)\right. \\
& \left.+(A+1)\left(\mu\left(\frac{c-a-b}{(a-1)(b-1)}-1\right)-\frac{c-a-b}{(a-1)(b-1)}\right)\right] \\
& \quad \leq(B-A)\left(\frac{1}{2(1-\beta) \cos \eta}+1\right)-\frac{(1-\mu)(A+1)(c-1)}{(a-1)(b-1)}
\end{aligned}
$$

is satisfied, then $I * f \in K(A, B)$.

Proof. The proof follows from Lemma 2.1 and by applying similar method as in the proof of Theorem 2.8; we omit the details involved.

If we let $\mu=0, A=2 \alpha-1$, and $B=1$ in Theorem 2.11, we get the following corollary.

COROLlary 2.12. Let $a>1, b>1$, and $c>a+b+1$. If $f \in R_{\eta}(\beta)$ and the inequality

$$
\begin{aligned}
& \frac{\Gamma(c) \Gamma(c-a-b)}{\Gamma(c-a) \Gamma(c-b)}\left[1-\frac{\alpha(c-a-b)}{(a-1)(b-1)}\right] \\
& \quad \leq(1-\alpha)\left(\frac{1}{2(1-\beta) \cos \eta}+1\right)-\frac{\alpha(c-1)}{(a-1)(b-1)}
\end{aligned}
$$

is satisfied, then $\int_{0}^{z}{ }_{2} F_{1}(a, b, c ; t) d t * f \in K(\alpha)$.

If we let $\alpha=0, \beta=0$, and $\eta=0$ in Corollary 2.12, we have the following corollary.

COROLlary 2.13. Let $a>1, b>1$, and $c>a+b+1$. If $f \in S$ and the inequality

$$
\frac{\Gamma(c) \Gamma(c-a-b)}{\Gamma(c-a) \Gamma(c-b)} \leq \frac{3}{2}
$$

is satisfied, then $\int_{0}^{z}{ }_{2} F_{1}(a, b, c ; t) d t * f \in K$.

ACKNOWLEDGMENT. This work was supported by the Korea Science and Engineering Foundation (KOSEF), project no. 2000-6-101-01-2.

\section{REFERENCES}

[1] Ju. E. Hohlov, Operators and operations on the class of univalent functions, Izv. Vyssh. Uchebn. Zaved. Mat. 10(197) (1978), 83-89 (Russian).

[2] W. Janowski, Some extremal problems for certain families of analytic functions. I, Ann. Polon. Math. 28 (1973), 297-326.

[3] S. Kanas and H. M. Srivastava, Linear operators associated with $k$-uniformly convex functions, Integral Transform. Spec. Funct. 9 (2000), no. 2, 121-132. 
[4] N. Shukla and P. Shukla, Mapping properties of analytic function defined by hypergeometric function. II, Soochow J. Math. 25 (1999), no. 1, 29-36.

[5] H. M. Srivastava and S. Owa (eds.), Current Topics in Analytic Function Theory, World Scientific Publishing, New Jersey, 1992.

[6] J. Stankiewicz and L. Trojnar-Spelina, Some parametric family of functions, Zeszyty Nauk. Politech. Rzeszowskiej Mat. Fiz. 14 (1992), 45-54.

[7] J. Stankiewicz and J. Waniurski, Some classes of functions subordinate to linear transformation and their applications, Ann. Univ. Mariae Curie-Skłodowska Sect. A 28 (1974), 85-94.

J. A Kim: Department of Mathematics, Pohang University of Science Technology, Pohang, Kyungbuk 790-784, Korea

E-mail address: jiakim@postech.ac.kr

K. H. Shon: Department of Mathematics, College of Natural Sciences, Pusan National University, Pusan 609-735, Korea

E-mail address: khshon@hyowon. pusan.ac.kr 


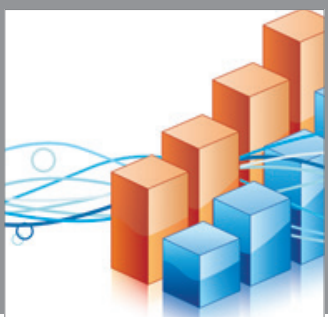

Advances in

Operations Research

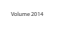

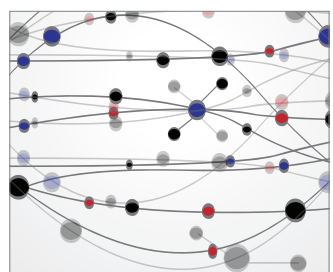

\section{The Scientific} World Journal
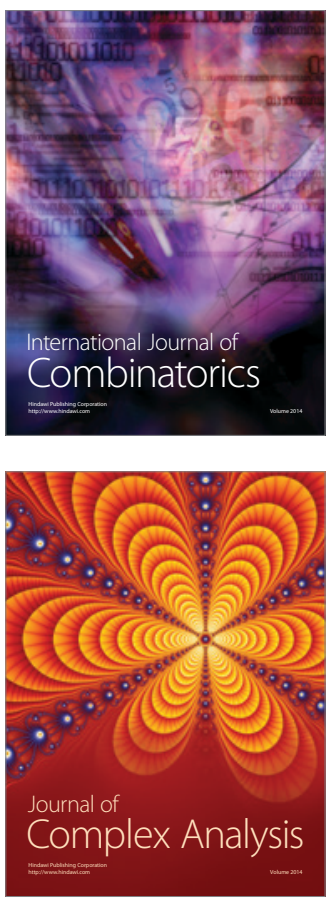

International Journal of

Mathematics and

Mathematical

Sciences
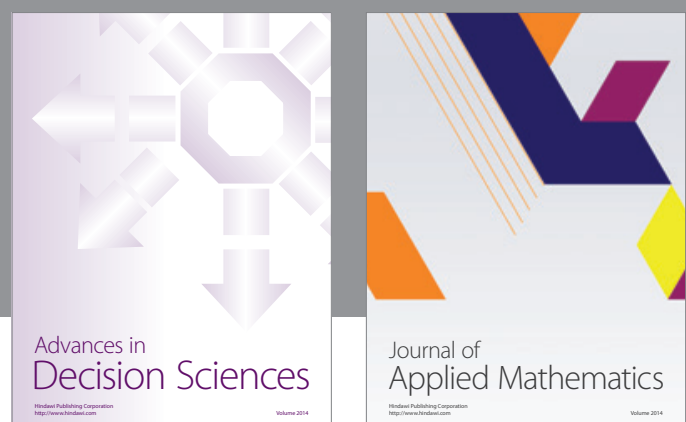

Journal of

Applied Mathematics
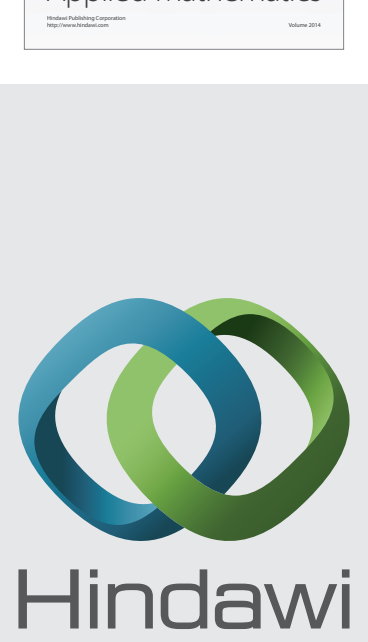

Submit your manuscripts at http://www.hindawi.com
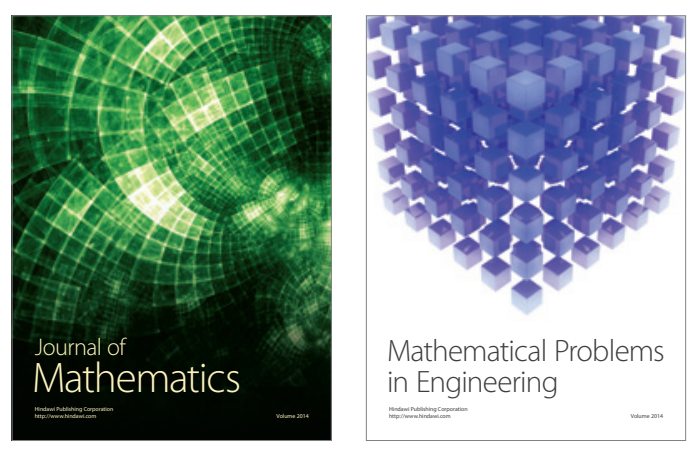

Mathematical Problems in Engineering
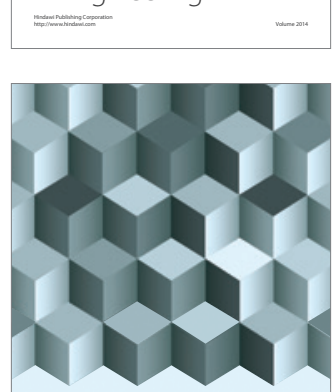

Journal of

Function Spaces
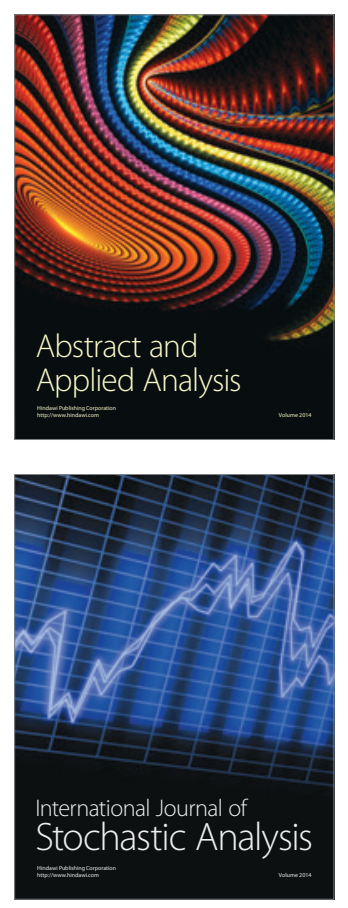

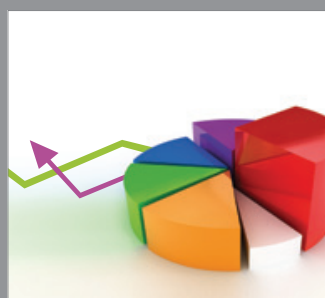

ournal of

Probability and Statistics

Promensencen
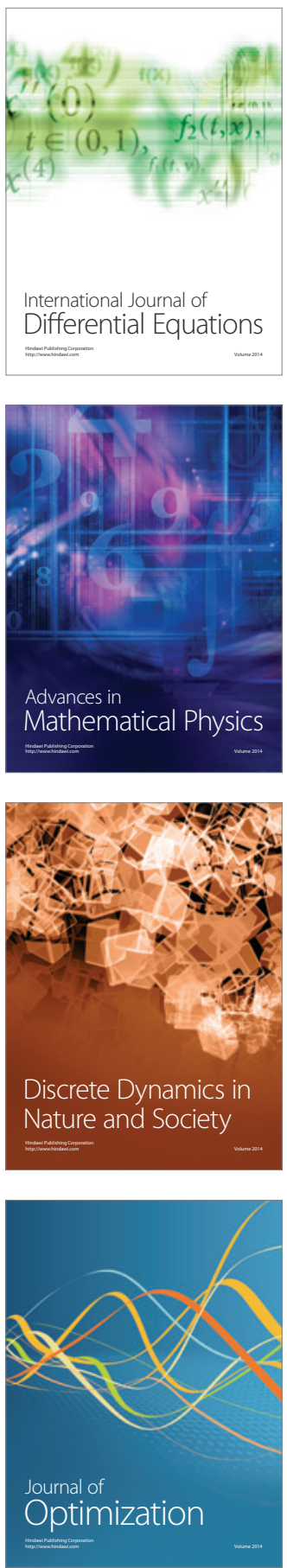